\title{
Meteor fluxes and visual magnitudes from EISCAT radar event rates: a comparison with cross-section based magnitude estimates and optical data
}

\author{
A. Pellinen-Wannberg ${ }^{1}$, A. Westman ${ }^{1}$, G. Wannberg ${ }^{2}$, K. Kaila ${ }^{3}$ \\ ${ }^{1}$ Swedish Institute of Space Physics, P.O. Box 812, S-981 28 Kiruna, Sweden \\ 2 EISCAT Scientific Association, P.O. Box 812, S-981 28 Kiruna, Sweden \\ ${ }^{3}$ University of Oulu, Department of Physical Sciences, Linnanmaa, FIN-90570 Oulu, Finland
}

Received: 15 October 1997 / Revised: 29 June 1998 / Accepted: 2 July 1998

\begin{abstract}
Incoherent scatter radars (ISR) are versatile instruments for continuous monitoring of ionisation processes in the Earth's atmosphere. EISCAT, The European Incoherent Scatter facility has proven effective also in meteor studies. The time resolution of the radar can be reduced to a few milliseconds, sufficient to resolve the passage of individual meteors through the narrow ISR beam. Methods for group and phase velocity determination of the meteoroids and the discrepancy between the results related to the target behaviour are presented. The radar cross sections of echoes associated with moving meteoroids ("meteor head echoes") are very small and increase with decreasing wavelength. The parent meteoroids are found to have visual magnitudes far below the detection limit of most optical observations. The equivalent visual magnitude limit of the smallest objects observed by EISCAT in the current experiments has been estimated by two different methods, both from the cross-section measurements and from the measured event rates. Both methods give a limit value of +10 for the smallest objects while the upper limit is +4 . The lower limit of the visual magnitude for the collocated optical measurement system is +4 . Thus the two detection systems observe two different meteor size ranges, with the radar almost reaching micrometeorite population. Meteor fluxes estimated from the event rates and the radar system parameters agree well with previous extrapolated values for this size range.
\end{abstract}

Key words. Ionosphere (ionization mechanisms). Radio science (ionospheric physics). Space plasma physics (ionization processes)

\section{Introduction}

In the early 1960s, a series of meteor studies were performed at the $440 \mathrm{MHz}$ Millstone Hill radar (Evans, 1965, 1966). This field of use of incoherent scatter radars then lay dormant for years, but has recently been revitalized. Since 1994, measurements have been performed at the Jicamarca, EISCAT and Arecibo incoherent scatter radars (ISR) and numerous papers have been published (Chapin and Kudeki, 1994; Pellinen-Wannberg and Wannberg, 1994, 1996; Zhou et al., 1995; Wannberg et al., 1996; Malnes et al., 1996; Mathews et al., 1997; Zhou and Kelley, 1997). Earlier studies comparing visual and in that case meteor radar observations on meteor head echoes have been done by Jones and Webster (1991).

Incoherent scatter radars usually operate at much higher frequencies than meteor radars. EISCAT uses $930 \mathrm{MHz}$ (UHF) and $224 \mathrm{MHz}$ (VHF). The VHF radar, located in Tromsø in Norway is monostatic, while the UHF radar is tristatic with the transmitter and one receiver located in Tromsø and the other receivers at Kiruna, Sweden and Sodankylä, Finland. In their standard mode of operation, these radars observe the incoherent scatter from collective density fluctuations, organized by plasma waves created at thermal nearequilibrium conditions in the ionosphere (Evans, 1969; Farley, 1979). By recording power spectra of the backscattering from these waves, an ISR can monitor basic plasma parameters such as electron density, electron and ion temperatures and plasma drifts. Other quantities, like collision frequency, mean ionic mass, electric field vector and some neutral atmosphere parameters can also be deduced. The vector drift velocity can be measured only at the tristatic intersection point, while all other parameters are measured along the whole radar transmitter beam. While the altitude range covered in this way ranges from some 70 to several hundred kilometers, the horizontal extent of the measuring volume is defined by the radar-antenna beam width and is quite narrow. For 
instance, the half-power opening angle of the EISCAT UHF radar is $0.6^{\circ}$ and that of the VHF $1.2^{\circ} \times 1.7^{\circ}$, resulting in beam diameters of about $1 \mathrm{~km}$ and $2-3 \mathrm{~km}$ respectively at $100-\mathrm{km}$ altitude.

In this paper we will give an overview of the methods developed for observing meteors by ISRs and summarize the work performed so far at EISCAT. We first describe the development of our meteor experiments and present hourly meteor rates for all our campaigns up to date. We proceed to briefly describe the meteoroid velocity estimate method, because an understanding of it is required for the estimation of effective radar cross sections of high Doppler echoes. Finally, we discuss the results from a comparison of simultaneous radar and collocated optical observations. Equivalent visual magnitudes of our radar events are estimated, and from these and the radar operating parameters a meteor flux estimate is computed.

The meteor project at the EISCAT radar facility began as a study of wintertime sporadic $E$ layers $\left(E_{\mathrm{s}}\right)$, which are associated with concentrations of heavy metallic ions, believed to originate from meteoroids. In Fig. 1 the current view of the whole process, from meteor impact to the very thin and dense ionized layers containing both $\mathrm{Na}$ and $\mathrm{Fe}$ positive ions, is shown in cartoon fashion. It is not yet well understood how the layers form. At times even neutral $\mathrm{Na}$ and Fe layers have been observed simultaneously by lidars (Clemesha et al., 1978; Clemesha, 1995). Since we wanted to resolve such very narrow structures in our radar measurements, a special, very sensitive high-resolution measurement scheme was designed for this study. The range resolution was set at $450 \mathrm{~m}$, which required the use of $3 \mu$ s pulses and $2 \times 176.8 \mathrm{kHz}$ detection band width in the radar receiver. With such a wide passband, also strongly Doppler shifted "meteor head echoes" were passed by the filters, and not cut away as they would be in most standard incoherent scatter program modes, where the detection band widths are of the order of $25 \mathrm{kHz}$.

\section{The experiments and the observations}

The radar observations reported in this paper were performed in December 1990 and December 1991, in association with the Geminid meteor shower (the Geminid campaigns), and in August 1993 during the Perseid meteor shower (the Perseid campaign). The Geminids had their maximum in 1990 on 14 December at 03 UT, and in 1991 on 14 December at 09 UT. The radiant transit occurred at about 01 UT. The Perseid shower was expected to be unusually active, since its parent comet, Swift-Tuttle (which is long-periodic with about 130 years period), had passed the Earth's orbit in December 1992. During the Geminid measurements, $66 \mathrm{~h}$ of EISCAT data were collected, while during the Perseids $11.5 \mathrm{~h}$ of UHF and $10.5 \mathrm{~h}$ of VHF data were collected. Overview descriptions of the experiment schemes used for these measurements are given in Pellinen-Wannberg and Wannberg (1994).

Figure 2 shows how meteor echoes and simultaneous weak auroral activity appear in a raw electron density vs. time series plot. At (00.29 UT, 00.30 UT, 00.31 UT, 00.34 UT, 00.36 UT, 00.39 UT) meteors show up as sharp, instantaneous peaks spread over an altitude range of about $70-130 \mathrm{~km}$, while auroral particle injections, even transient ones, (e.g. at 00.23 UT, 00.35 UT, 00.38 UT) last longer and ionize also at higher altitudes. Figure 3 shows some examples of meteor head echoes as they appear in Barker coded raw electron density profiles. They have very strange and unphysical forms, the cause of which will be explained in the next section. These echoes have now been proven to last only a few tens of milliseconds, the length of time it takes a meteoroid to pass through the radar beam. Figure 4 shows an example of events that are interpreted as trail echoes. These echoes never show splitting and can last for many seconds, i.e. as long as it takes for the meteor trail drifting with the ionospheric winds to pass through the radar beam. Figure 5 shows average hourly meteor rates for different periods from all the Geminid campaigns. Head and trail echoes have been separated, while Fig. 6 shows hourly UHF and VHF meteor head echo rates for the Perseid campaign. All the data show quite constant meteor rates, regardless of whether the measurements were made outside shower periods or during showers. The first measurement sessions run at the beginning of December 1990 (Fig. 5a) and 1991 (Fig. 5d), and the third one, about a week after the shower in 1990

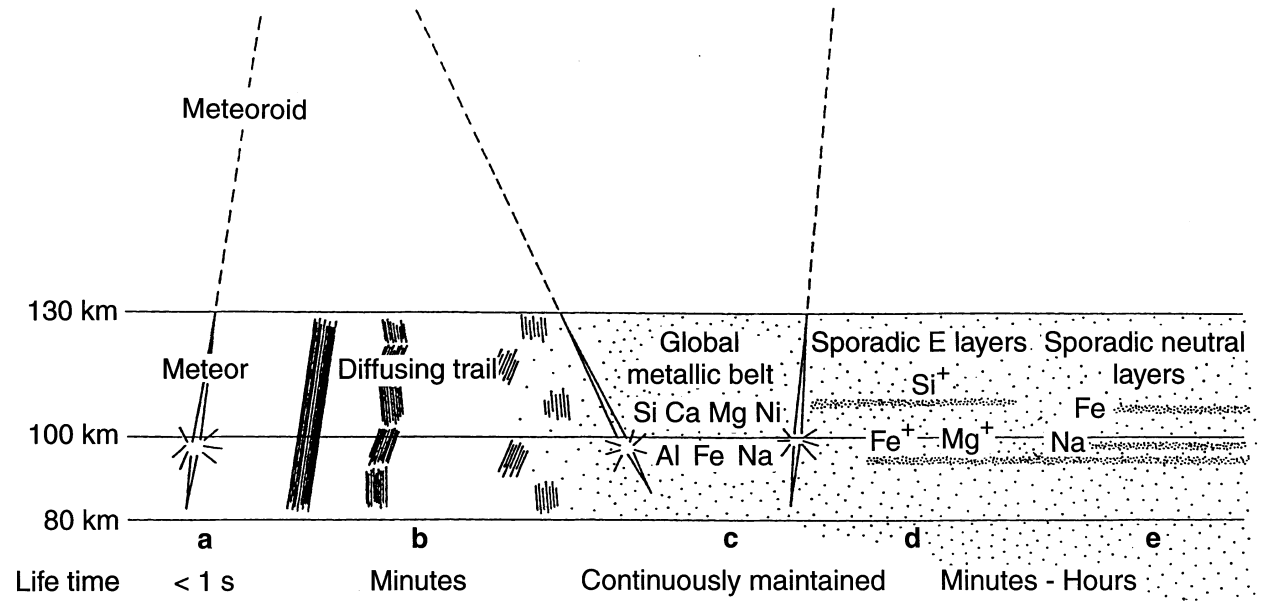

Fig. 1. Illustrating the process of meteoroid deposition in the atmosphere. The visible meteor is instantaneous (a), while its trail can be observed for minutes (b). The global metallic belt is continuously maintained by deposition of meteoric matter (c), while the old constituents slowly drift downwards and are lost. Ionised sporadic $E$ layers are formed occasionally (d) and sporadic neutral layers can form simultaneously (e). These layers can stay from minutes up to several hours 
(Fig.5 c), should have been free of any Geminid shower related meteor activity, but still show almost as high rates, close to 10 echoes $\mathrm{h}^{-1}$, as the measurements taken during the most active days of the Geminids between 12 and 15 December (Figs. 5b, e). It should be pointed out in this context that the radar pulse pattern used in the before-the-shower measurement session (Fig. 5a) had only half the power profile duty cycle used in all later runs. It therefore suffers from a $41 \%$ worse signal-tonoise ratio and a correspondingly higher detection threshold, and shows lower average event rates.

In the Perseids case, event rates from two days before the expected shower maximum and from the maximum night are not remarkably different, see Fig. 6 showing both UHF (Fig. 6a, b) and VHF (Fig. 6c, d) data from identical program modes. These data were taken eight months after the shower parent comet had passed through the central solar system in December 1992, and the shower was expected to become unusually stormy. However, neither optical (Brown and Rendtel, 1994) nor radar observations observed any storm level activity. The shower maximum was predicted to occur between
22 UT, 11 August and 03 UT, 12 August (Rao, 1993), as the Earth would cross the comet orbit plane at 01:15 UT. The UHF data (Fig 6. b) actually show increased rates towards the end of the measurement period, in agreement with optical observations (Brown and Rendtel, 1994), but in the VHF observations the meteor rates were higher two days earlier (Fig 6.c) and were decreasing during the expected maximum period (Fig 6.d).

In the Geminid data set, in Fig. 5 about $75 \%$ of the observed events are head echoes. In the simultaneous UHF and VHF Perseids measurements the data has so far only been scanned for head echoes (Fig. 6). Many more events were observed at VHF than at UHF, but this is probably mostly due to the much wider field of view of the VHF antenna. Even after accounting for this, it is difficult to estimate the normalized meteor rates, as there is also the scattering cross-section frequency dependence we have reported in Wannberg et al. (1996) due to the scattering mechanism at these frequencies. The average hourly rates over all our measurements on the UHF radar are bout 8 and on the VHF radar about 65 meteor head echoes.

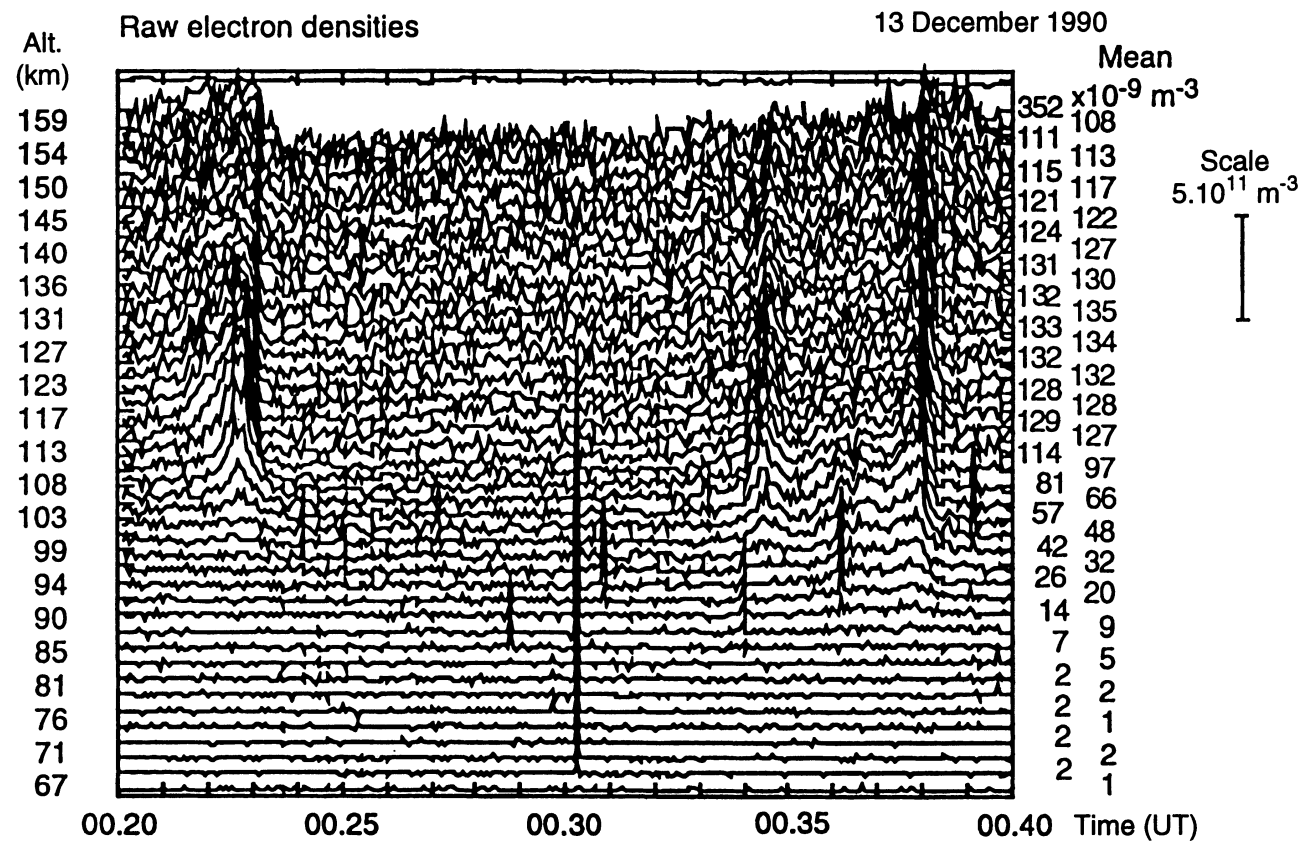

Fig. 2. EISCAT-UHF raw electron density time series versus altitude showing both meteors and weak auroral activity. The time series show the instantaneous raw electron densities of the ionosphere at each gate. The mean values over the 20 -min periods are shown in the righthand column. The vertical line at the right shows the dynamical range of the densities

Table 1. Optical meteors observed at Tromsø

\begin{tabular}{lllll}
\hline Day & Observation time $[\mathrm{h}]$ & Geminids & Sporadic meteors & Notes \\
\hline $10-11$ December & 4 & 4 & 2 & - \\
$11-12$ December & - & - & - & cloudy \\
$12-13$ December & $1-2$ & 3 & - & aurora \\
$13-14$ December & - & - & - & cloudy \\
$14-15$ December & 6.2 & 49 & - & - \\
$15-16$ December & - & - & - & cloudy \\
$16-17$ December & 0.3 & - & 3 & - \\
Total & 12 & 56 & & \\
\hline
\end{tabular}


Table 2. Optical meteors observed at Esrange

\begin{tabular}{lllll}
\hline Day & Observation time $[\mathrm{h}]$ & Geminids & Sporadic meteors & Notes \\
\hline $10-11$ December & 11 & 1 & 3 & - \\
$11-12$ December & - & - & - & cloudy \\
$12-13$ December & 2 & 2 & - & aurora \\
$13-14$ December & - & - & 2 & cloudy \\
$14-15$ December & 9.2 & 19 & 3 & - \\
$15-16$ December & 11 & 4 & 1 & aurora \\
$16-17$ December & 2 & 0 & 9 & - \\
Total & 35.2 & 26 & & \\
\hline
\end{tabular}

Power profiles
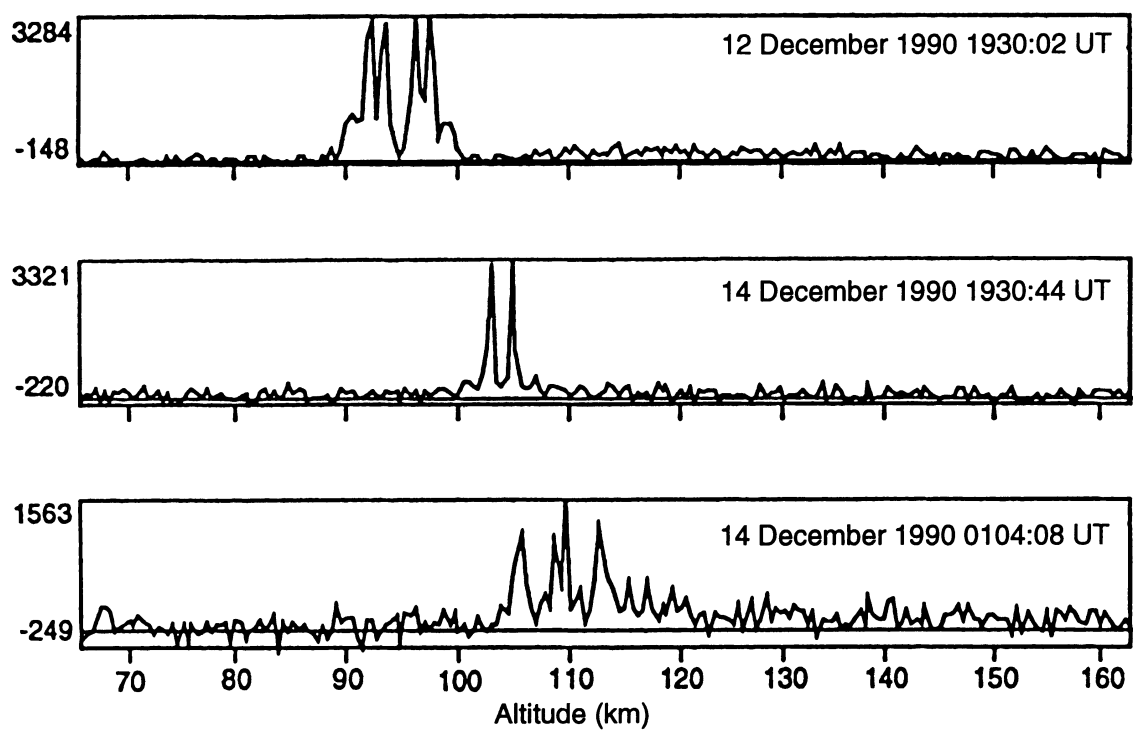

Fig. 3. 2-s integrated raw electron density altitude profiles containing meteor head echoes from the EISCAT UHF radar. The vertical axis give the dynamical range of the signal. The numbers at the upper and lower left corners of each panel refer to this range. The background noise level is subtracted from the signal. The horizontal lines in the lower part of each panel indicate the zero levels. The echoes are seen only in isolated 2-s dumps
The December 1990 Geminid campaign on EISCAT partly overlapped with a Finnish optical auroral campaign. Due to the very low auroral activity, the two low light level television cameras, located at Ramfjordmoen, close to the EISCAT Tromsø site and at Esrange, close to the EISCAT Kiruna site, could instead record many meteor streaks. These observations are summarized in Tables 1 and 2. Both cameras were of the ISIT-type with two image intensifiers. The camera used in Tromsø employs a telecentric system and a filter wheel with four positions, fitted with bandpass filters for different auroral spectral lines. One of the positions is empty and used for white light measurements, and thus applicable for meteor observations. During the campaign, most of the data was taken in this broadband mode, due to the low auroral activity. Data was recorded on VHS video tape and image time-stamped by a video timer. Fisheye optics giving a field of view of about $90^{\circ} \times 150^{\circ}$ was used, and the camera was pointed close to vertical. Due to the telecentric system the light throughput is not very high and the images are rather noisy. The camera used in Esrange has a wide angle lens, with a field of view of $50^{\circ} \times 75^{\circ}$. The image quality is higher than in the Tromsø camera. The camera was pointed towards the EISCAT radar beam at an azimuth of $160^{\circ}$ and an elevation of $42^{\circ}$.
The limiting magnitudes of the cameras were calibrated against known stars and found to be approximately +3.5 for the Tromsø and +4 for the Esrange camera. During the campaign, 59 meteor trails were recorded at Tromsø and 35 trails at Esrange. Many of them were observed by both cameras simultaneously. Out of the 59 events observed by the Troms $\varnothing$ camera, eight were found to pass close to the EISCAT radar beam. However, none of these optical meteors were simultaneously detected by the radar.

\section{The velocity estimate method}

We refer back to Fig. 3 and the strange power profile events. There are always spread over 25 gates, and they can by no means represent any physical ionization distributions in the ionosphere. In Wannberg et al. (1996) we have shown that the forms are consequences of the Barker-coding of the radar pulses. The ambiguity function of the used 13 baud Barker code spreads over $2 \times 13-1=25$ gates. In ordinary ionospheric measurement modes, the central gate contains almost all the signal power. However, at higher Doppler shifts the signal power spreads into the other gates (see Fig. 6 in Pellinen-Wannberg and Wannberg, 1994). The decoding 
Power profiles
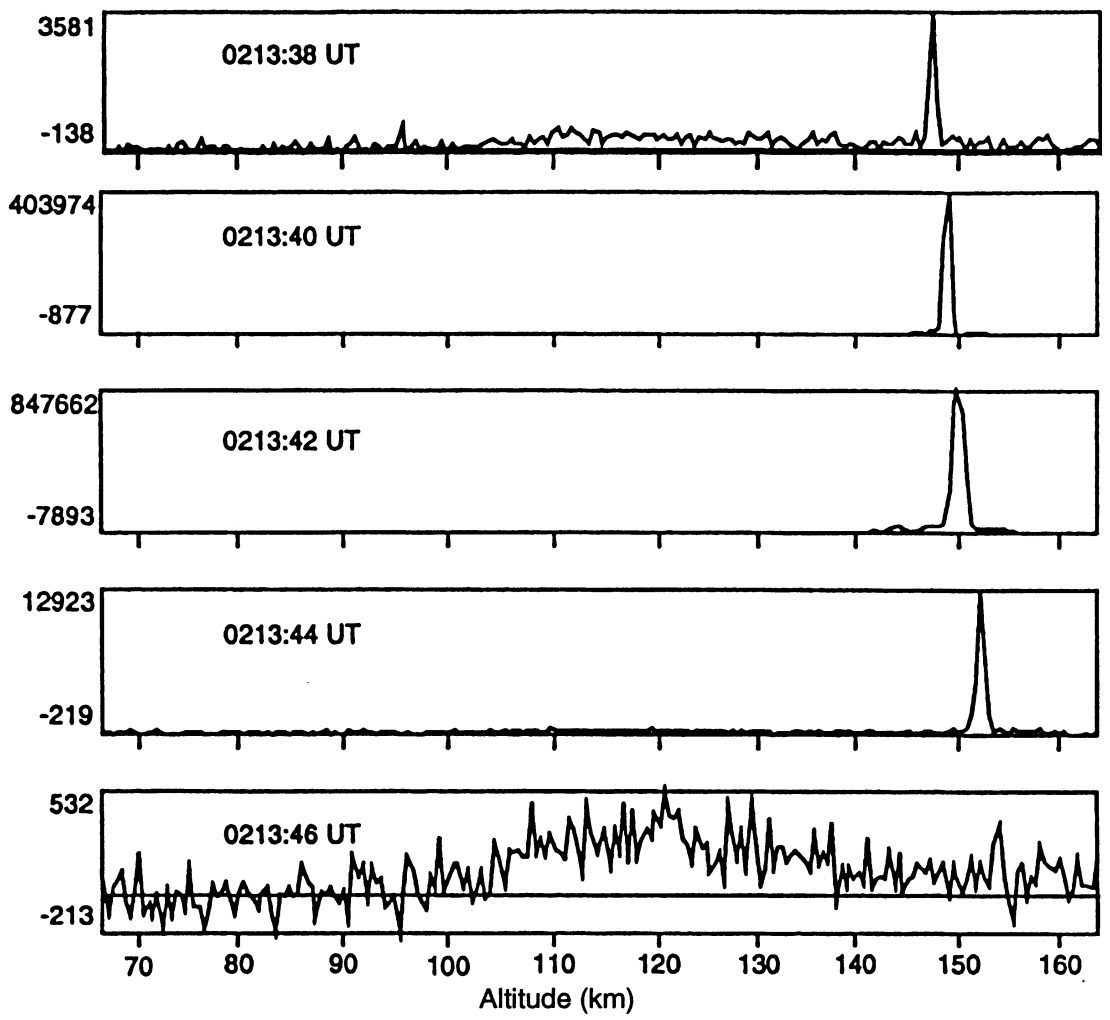

Fig. 4. As Fig. 3, but showing meteor trail echoes from an ionized trail left behind a meteor from the EISCAT UHF radar. This echo can be detected in several dumps
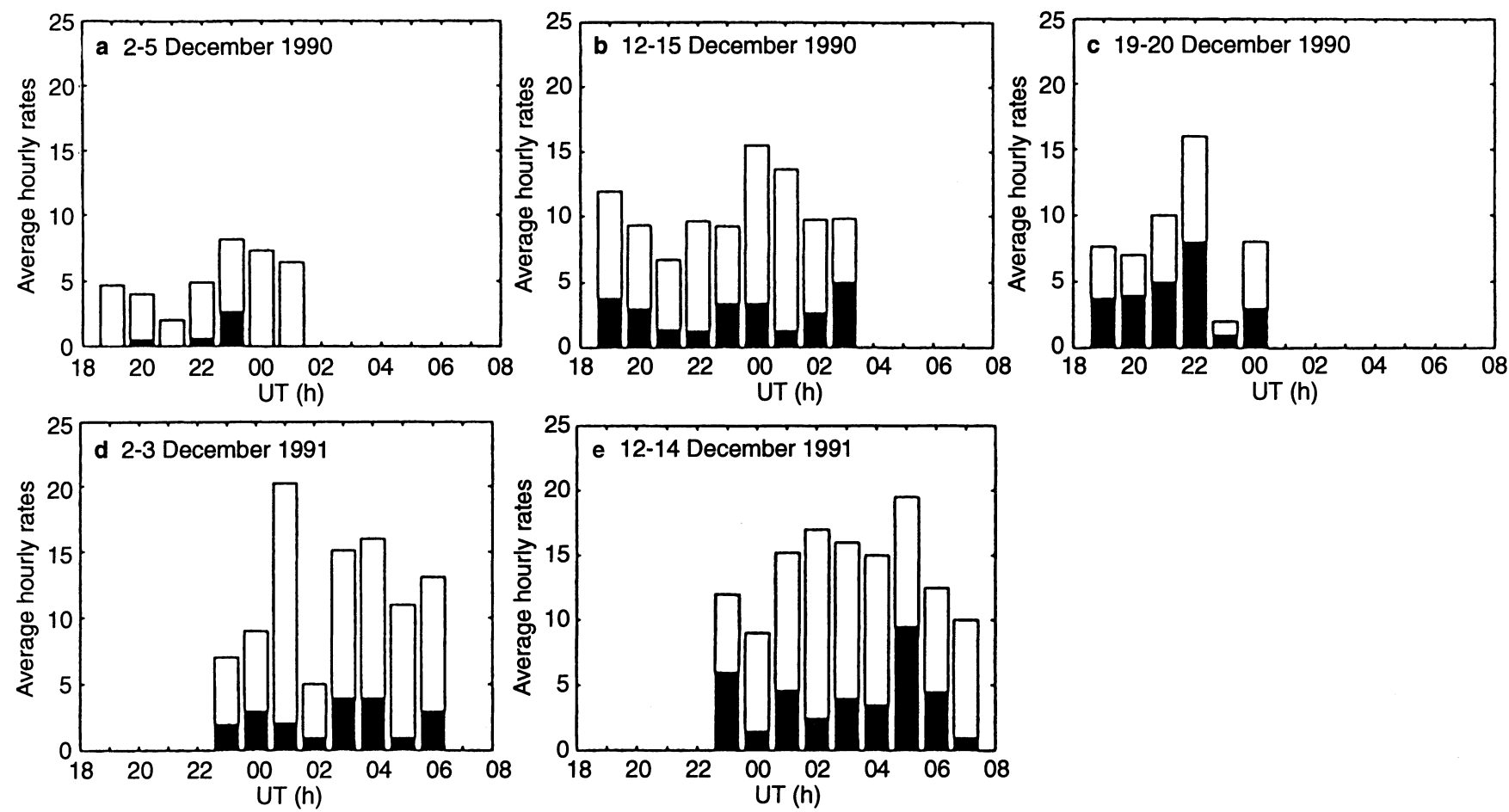

Fig. 5. Average hourly meteor trail (black) and head (white) echo rates from different periods of the Geminid campaigns in 1990 and 1991 . The data are taken with the UHF radar.

of the Barker-compressed signal on receive is performed by matched filtering techniques. If the scattered signal is Doppler shifted, it becomes detuned relative to the filter, and the target response then spreads over all the 25 gates in the 13-baud Barker-code case. Figure 7 shows how the response varies as a function of target velocity. 

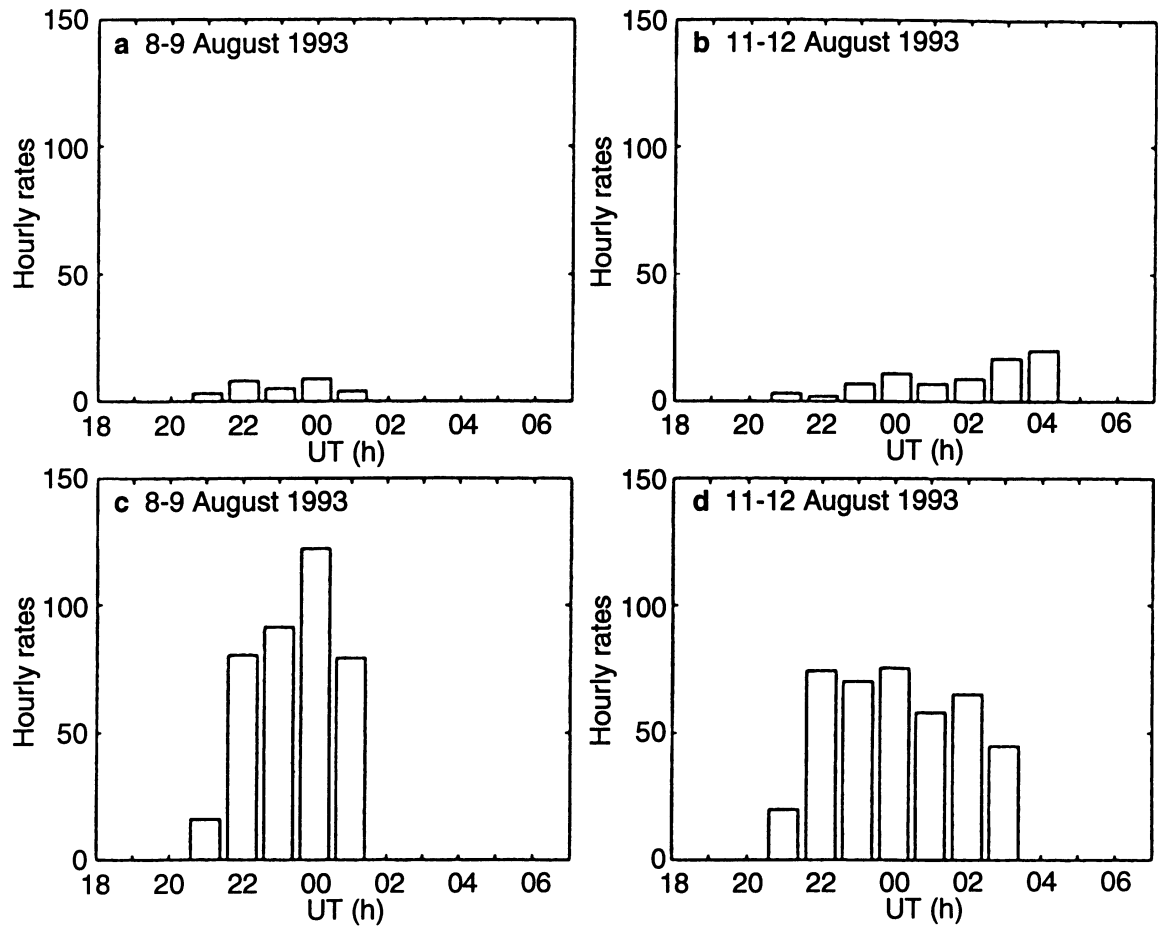

Fig. 6. Hourly rates of the Perseid campaign in August 1993. The data are taken simultaneously with the UHF $(\mathbf{a}, \mathbf{b})$ and the VHF (c, d) radars
Since the splitting of the signal is a function of the Doppler shift, it can be used for accurate target velocity estimates. Figure 8 shows an example of velocity fitting. As the ambiguity function is rapidly varying, very good data are required. This is most often also fulfilled for the EISCAT UHF radar, while the data from the VHF system are generally more noisy (see Fig. 10 in Wannberg et al., 1996). The event of Fig. 8 is from a single $2 \mathrm{~s} \mathrm{UHF}$ data dump from the Geminid campaign. No signal is seen in the dumps immediately before, or after, this one, and the duration of this echo is therefore certainly less than 2 s. From fitted and previously known Geminid meteor- oid velocities, we could estimate that at $100-\mathrm{km}$ altitude, meteoroids would pass through the EISCAT UHF radar beam in a few tens of milliseconds at most. A time-sliced data-taking procedure, providing $6 \mathrm{~ms}$ time resolution, was therefore introduced for the Perseid measurements in order to time resolve the head echo events (Wannberg et al., 1996). Figure 9 shows how a meteoroid passes the radar beam at an oblique angle and a Doppler line-ofsight speed of about $27 \mathrm{~km} \mathrm{~s}^{-1}$.

The time-resolved data can be used to determine average meteor line of sight velocities by the time-offlight method, which can be compared to the fitted

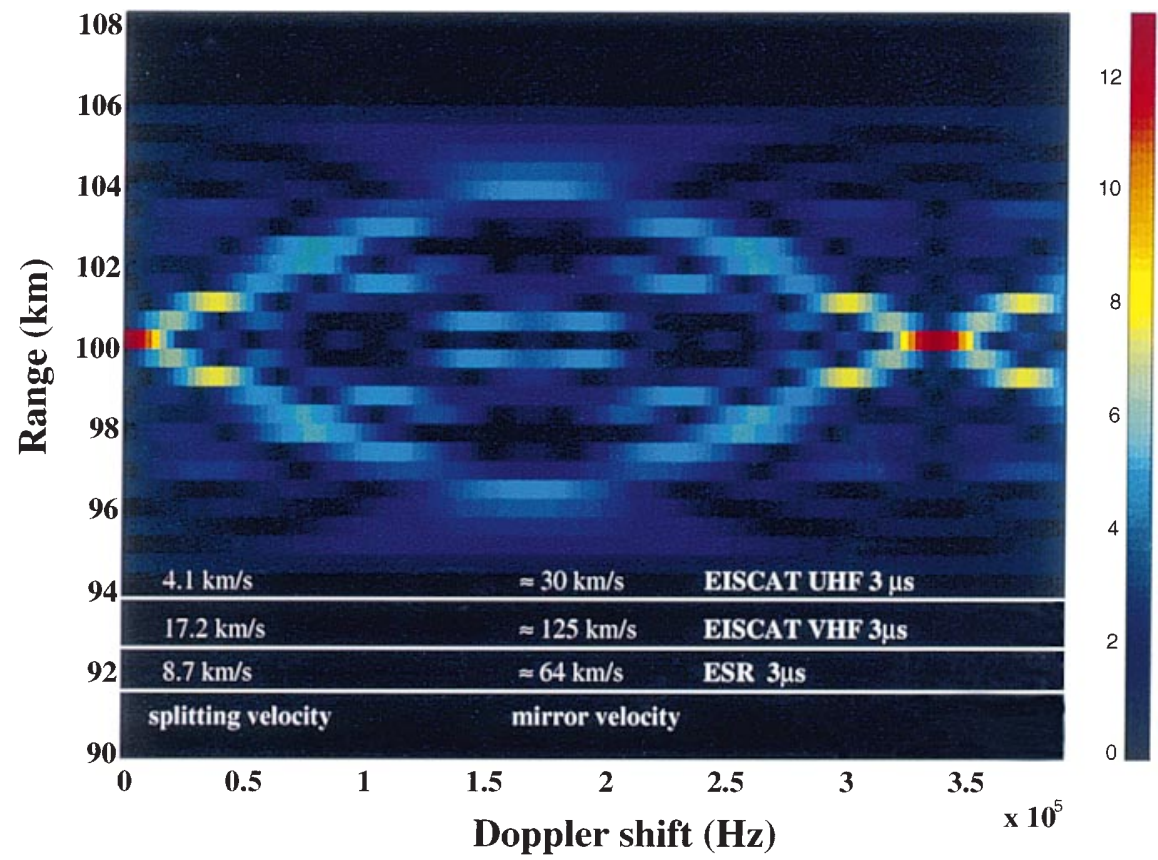

Fig. 7. The power domain response of a matched-filter decoded radar echo from a moving point target, centered at $100 \mathrm{~km}$ range and illuminated by a $13 \times 3 \mu$ s Barker code. The splitting velocity is the velocity at which visible splitting of the ordinary ambiguity function can first be detected. The response shape mirrors at the so-called mirror velocity. Splitting and mirror velocities for the EISCAT UHF, VHF and Svalbard radars are indicated 


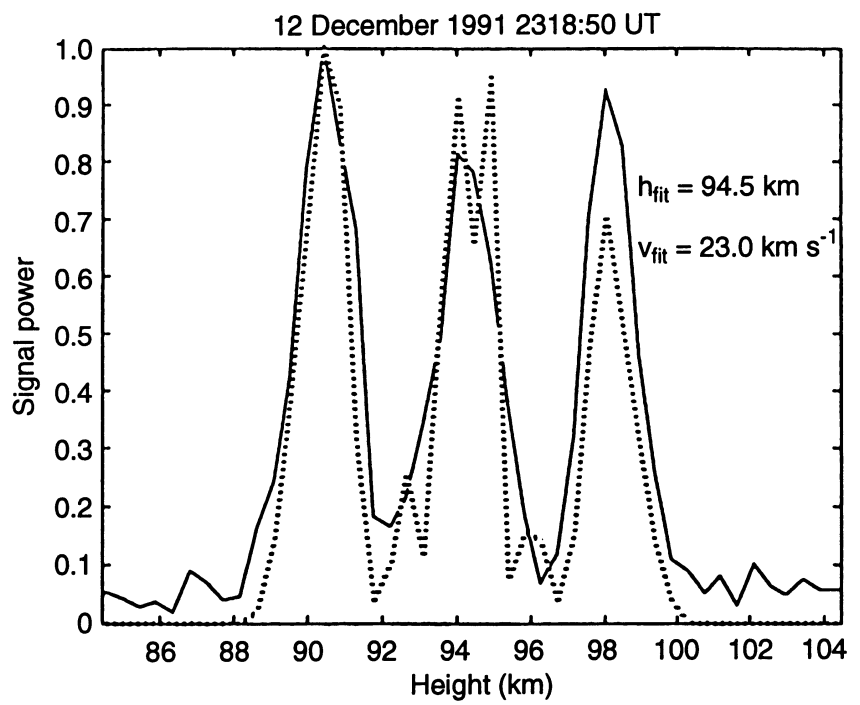

Fig. 8. An example of a velocity fit on a typical head echo event from the Geminid campaign in 1991. The measured data is shown in full line and the model fit in dashed line

Doppler velocities. With the time-of-flight method we simply estimate the velocity by dividing the altitude difference at the highest and lowest points the meteor was observed with the time difference between these observations. The event of Fig. 9 shows a strange effect which has been observed in a large percentage of all such resolved events: the time-of-flight estimated target group speed is about $33 \mathrm{~km} \mathrm{~s}^{-1}$, that is, significantly greater than the speed value obtained from the Doppler fit! If this latter velocity is understood as the effective phase velocity of the scatterer, this observation seems to indicate that the scattering occurs from a plasma which is slipping relative to the parent meteoroid at a speed equal to half the difference between the group and phase velocities. This phenomenon is the subject of an ongoing investigation and will be addressed in a separate paper.

More important in the context of the present paper is the fact that the total signal power is a conserved quantity, represented by the area under the ambiguity function, even when this is spread out (Wannberg et al., 1996). The effective radar cross sections of Dopplershifted head echoes can therefore be estimated from the received signal power. We pointed out earlier that it is the use of a wide receiver passband which makes the observation of frequency-shifted head echoes possible. However, as the Doppler shift increases, less and less of the signal power eventually falls within the filter. This effect is described in detail in the Appendix of Wannberg et al. (1996), and shown in their Fig. A2. Thus the total received power decreases for signals from very fast targets. This must be compensated when making crosssection estimates from the apparent received power.

\section{Target cross-sections and magnitudes}

Early measurements performed with high power UHF radars (Evans, 1965) were made by looking normal to the radiants of some major showers at very low antenna elevation angels. Thus the meteor-atmosphere interaction volumes were at long ranges (about $800 \mathrm{~km}$ ). Even so, about 40 echoes $\mathrm{h}^{-1}$ were seen on the average. Starting from this observed rate, it was estimated that these echoes corresponded to a visual meteor magnitude of +8 . The scattering cross sections of the targets were estimated at around $1 \mathrm{~m}^{2}$, which is much larger than the cross sections seen in the recent Arecibo and EISCAT

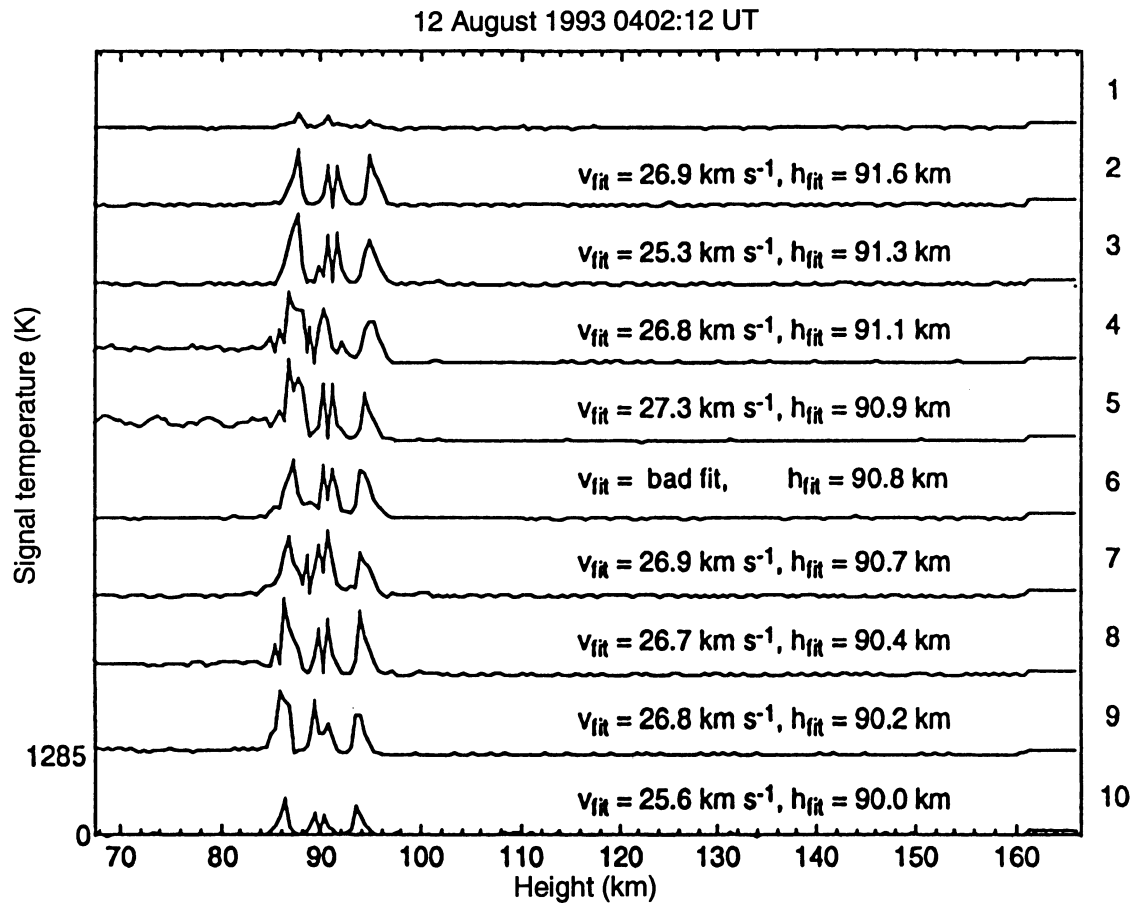

Fig. 9. A. Perseid-time head echo resolved in $6 \mathrm{~ms}$ time-slices. The meteoroid passed the radar beam within about $60 \mathrm{~ms}$. From such data the meteoroid descending process can be studied 


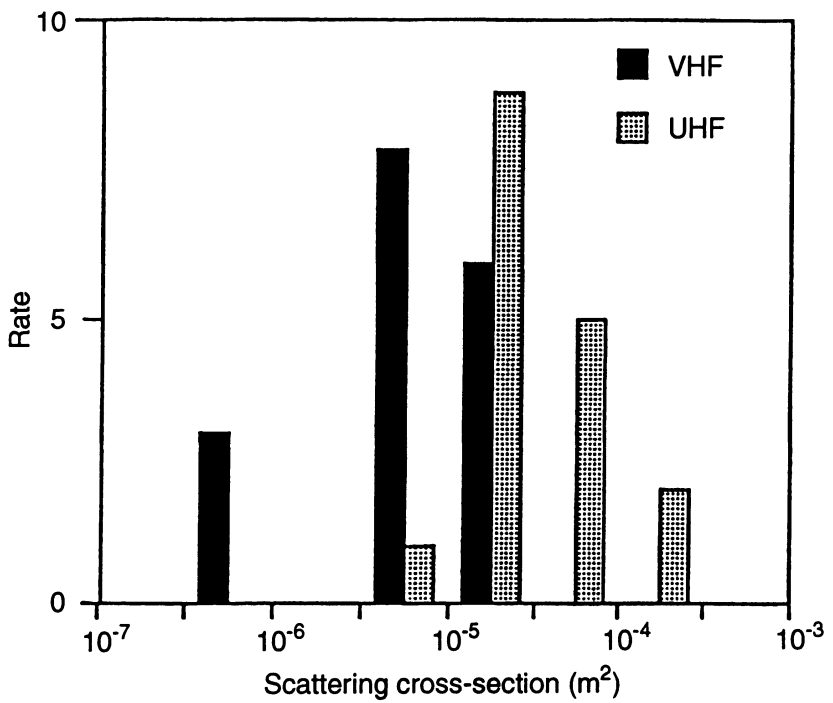

Fig. 10. Observed scattering cross-section histograms for 17 head echoes observed with both UHF and VHF radars during the Perseid campaign

measurements. At Arecibo, meteor echoes have been a problem in connection with the regular E layer incoherent measurements and great efforts have been made to remove them (German and Mathews, 1986; Zhou and Mathews, 1994). A study of incoherent radar data taken in 1991 and 1992 (Zhou et al., 1995) showed an observed flux of about 25 meteors $\mathrm{h}^{-1}$, corresponding to visual magnitude of +14 . These measurements were time integrated and thus no velocity or spectral information was available. Later, dedicated meteor measurements with the Arecibo radar (Mathews et al., 1997) with direct high speed storage of the sampled receiver output signals have made it possible to study the meteor head echoes in greater detail both in time and space. These measurements have a range resolution of $150 \mathrm{~m}$ and a time resolution of $1 \mathrm{~ms}$ over a height range of 93-102 $\mathrm{km}$. The observed meteor rate corresponds to objects with a visual magnitude of +15 and having scattering cross section $1 \times 10^{-8} \mathrm{~m}^{2}$, which should correspond to particles with masses about $1 \mu \mathrm{g}$, if the classical meteor rate vs. mass distribution is applicable (Hughes, 1978).

Wannberg et al., (1996) discuss how target cross sections can be estimated from EISCAT data. Scattering cross-sections estimated as briefly described in sect. 3 range between $7 \times 10^{-6}-2 \times 10^{-4} \mathrm{~m}^{2}$ for the UHF and $5 \times 10^{-7}-3 \times 10^{-5} \mathrm{~m}^{2}$ for the VHF. Figure 10 shows the cross sections for the simultaneous VHF and UHF meteor head echoes. We have shown earlier that such cross-sections fall in the Rayleigh region corresponding to small targets (Wannberg et al., 1996), and postulated that the actual radar target is an overdense cloud (a "ball") of plasma contained within the meteor coma, and not the solid meteoroid. Under this assumption, these plasma targets should have approximate diameters of $2.0-4.0 \mathrm{~cm}$ at the EISCAT UHF and $4.2-7.3 \mathrm{~cm}$ at the EISCAT VHF frequency.

Starting from the assumption that these target size estimates represent the approximate extent of the meteor coma, and further knowing the critical densities at $224 \mathrm{MHz}$ and $931 \mathrm{MHz}$, Westman (1997) has used a model based on ionisation in single collisions between the meteoroid body and the air molecules to calculate the corresponding meteor body sizes. These are found to fall in the range $0.1-1.0 \mathrm{~mm}$, corresponding to a range of visual magnitudes from about +10 to +4 . However, since this estimate hinges on the particular assumption about the scattering being from overdense plasma balls, the particle sizes are open to question until verified by independent means.

This has now been done. As shown in sect. 2, the EISCAT measurements yield average event rates of 8 meteors $\mathrm{h}^{-1}$ for the UHF and 65 meteors $\mathrm{h}^{-1}$ for the VHF system. The corresponding normalized fluxes have been computed, taking the radar beam patterns and modulation waveforms into account, and are found to be about 0.1 meteors $\mathrm{m}^{-2} \mathrm{y}^{-1}$ for both radars. These fluxes are true for meteors that spend more than the interpulse period, $6 \mathrm{~ms}$, in the radar beam, so that at least one complete Barker code sequence illuminates them. Under this condition the effective detection duty cycle is close to $100 \%$. Assuming that the measured flux is an average over all observable target sizes, and comparing it to established flux vs. magnitude distributions (Fig. 11; Sugar, 1963) it is found to correspond to an average magnitude of about +10.0 and a lower size cut-off at approximately magnitude +10.5 , in fair agreement with the +10 estimated from coma size. Thus two independent methods produce limiting magnitude estimates which agree to within the measurement uncertainties.

\section{Discussion}

Meteor studies using incoherent scatter radars promise to add significantly to our knowledge about the interaction of very small meteors with the upper atmosphere. ISRs use higher frequencies than typical

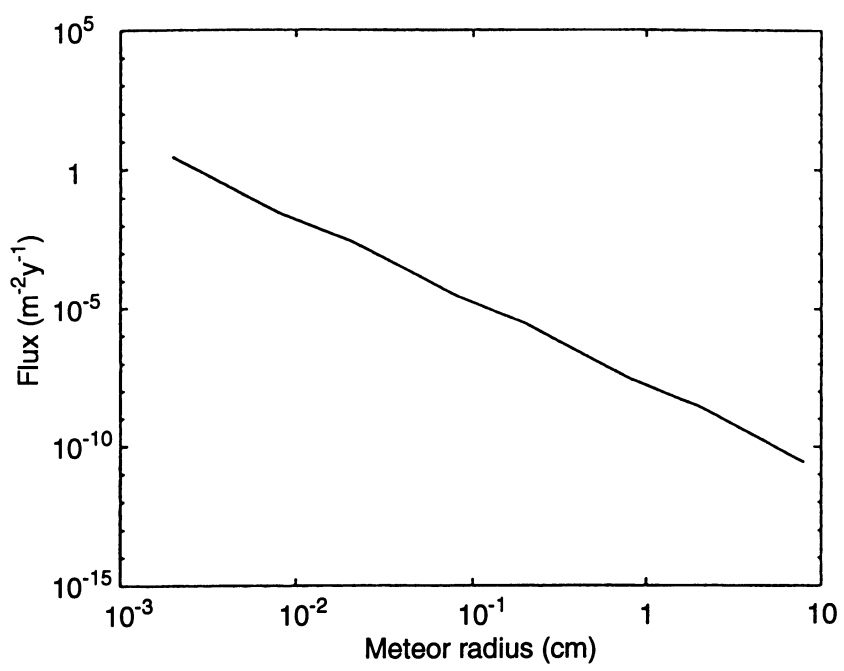

Fig. 11. Meteor flux as function of meteor radius at a height of $105 \mathrm{~km}$ 
normal incidence VHF meteor radars and their poweraperture products and receiver sensitivities are orders of magnitude greater, which allows the detection of very small targets that would be far below the detection threshold of the normal incidence systems. Recent experiments at the Arecibo and EISCAT radars have also shown a significant aspect insensitivity, i.e. meteors entering the beam from all directions can be observed. In addition, ISRs can monitor the state of the background ionosphere throughout the interaction region at all times. Perhaps the only important drawback of this method is that incoherent radar beam widths are very narrow - the halfpower opening angles are only a few degrees, compared to tens of degrees for conventional meteor radars. Thus only very small projected areas in the $E$ region can be monitored, which reduces the event rate and makes the determination of statistically significant solid angle flux distributions a tedious process.

The optical observations made during the 1990 Geminid shower did not produce any visual meteor event which could be correlated with a simultaneous event in the EISCAT radar data. We interpret this as being due to both the difference in sensitivities and the different fields of view of the instruments used. Whereas the optical instruments have a very large opening angle compared to the EISCAT radar systems, their limiting visual magnitudes are around +4 . Due to their large fields of view, the optical instruments can still register a reasonably high rate of magnitude +4 or brighter meteors. On the other hand, the EISCAT radars have an equivalent visual magnitude limit of +10 , but only register events that occur in a narrow column of about $1-\mathrm{km}$ diameter extending through the $E$ region. While it is true that the radars are largely aspect insensitive within this column, and thus can "see" meteors from the whole visible hemisphere, the limited size of the interaction volume results in low absolute event rates. Our present understanding is thus that the EISCAT radars observe a part of the meteor population different from that seen by either optical instruments or normal incidence radars, and consisting of smaller and more numerous objects. This seems to differ from the comparing visual and head echo observations on meteor radars. Jones and Webster (1991) report from their observations extending over 20 years at the Spring Hill Meteor Observatory that every visual meteor seems to produce a head echo which however may be too weak to be detected.

The presently accepted sporadic meteor flux vs. size distribution is such that the flux at magnitude +4 is about one hundred times less than at magnitude +10 . This means that statistically only one or two magnitude +4 meteors per day would pass through the EISCAT observation volume. Whether these can be observed anyway is a different matter.

The signal powers and deduced UHF and VHF cross-sections of all the observed radar head echoes fall into a surprisingly narrow range (Fig. 10). It is not obvious why this is so, since the meteoroid size distribution is expected to be continuous. Clearly, the global sensitivity of the radar system sets a lower detection limit, but the conspicuous absence of stronger echoes is much more difficult to explain. Even if the flux of magnitude +4 meteors is so low that they can almost be excluded on statistical grounds, there is still a range of particle sizes from magnitude +10 to at least magnitude +7 which are so numerous that several of them should have been observed during our measurements.

Our present best explanation of this observation is that it is a consequence of the fact that the properties of the gas flow around a meteor are governed basically by the ratio of particle size and gas mean free path. The echo-forming mechanism suggested by Westman (1997) depends on a combination of increased ionisation rate and compression of the interaction volume in front of a meteoroid to raise the electron density to above critical. For this mechanism to work, the meteor-gas interaction must take place in the so-called free molecular flow regime (Bronshten, 1983). If the particle size is increased at a given atmospheric mean free path, the interaction moves away from the free-molecular regime into a transitional regime where a part of the ablated meteor matter is continually slipping off the meteor coma and into the meteor trail. As defined by Bronshten (1983), a 1 -mm radius particle would remain in the free-molecular regime down to about $85-\mathrm{km}$ altitude. Of course, the boundaries between the different flow regimes are not hard, and it appears that some aspect of the target formation is very sensitive to plasma loss, thus possibly preventing the plasma density from ever reaching critical in the case of the larger particles.

Since the radar is sensitive to meteors entering the beam from all direction, it is impossible to deduce from a monostatic radar data set whether the detected events are associated with shower meteors or with the sporadic meteor population. But using the EISCAT UHF radar, the system can be run in tristatic mode and the remote receiver antennas directed to the altitude of maximum event rate in the $E$ layer, as determined by earlier monostatic measurements. This allows the determination of the full velocity vector and three independent scattering cross section estimates for every event in the common scattering volume. If the Barker code baud length is chosen appropriately (for instance decreased to $2 \mu \mathrm{s}$ ), and multiple frequency offset receiver channels are employed, it should even be possible to identify meteoroids from interstellar space with velocities above the solar system escape velocity, $72 \mathrm{~km} \mathrm{~s}^{-1}$ (Taylor et al., 1996), if there are any. An experiment employing these techniques will be operated for the first time during the November 1997 Leonids meteor shower.

Unfortunately, there are some down-to-earth difficulties associated with using ISRs for meteor observations. Incoherent scatter radars are very expensive to run. They usually operate only for a limited number of hours per year (in the case of EISCAT less than $2000 \mathrm{~h}$ in total). The competition for these hours is fierce. Besides, the meteor studies require advanced program modes, which are still under development, and the standard signal-processing systems used for ionospheric measurements are too limited for optimal meteor observations at most facilities. 
There are, however, compelling scientific reasons for continuing, and further developing, this technique, one of the strongest being the return of comet Tempel-Tuttle, the parent comet of the Leonid meteor shower, to the inner solar system in 1998. This will be covered by many measurements at the Arecibo and EISCAT radars and other instruments over 3-4 years, and a lot of exciting new results are to be expected.

\section{Conclusion}

The EISCAT VHF and UHF incoherent scatter radars have been operated in a mode optimized for the detection of Doppler shifted echoes from meteors. Data from three campaigns in 1990, 1991 and 1993 have been analyzed for meteor event rates and these have been converted to normalized meteor flux. The event rates are observed to be quite constant both during showers and outside shower times. They are on average $8 \mathrm{~h}^{-1}$ for UHF radar and $65 \mathrm{~h}^{-1}$ for the VHF radar. Both values correspond to a normalized flux of 0.1 meteors $\mathrm{y}^{-1} \mathrm{~m}^{-2}$. By referring to established flux vs. magnitude models, the average equivalent visual magnitude of the observed population is around +10 , with a lower cutoff at around magnitude +10.5 , in good agreement with the +10 detection limit magnitude independently determined from the particle sizes estimated by Westman (1997) from the measured effective target cross sections. An optical data set recorded during the 1990 campaign by a collocated wide angle system with a limiting magnitude of +4 has also been analyzed. Although eight events recorded by this system passed close to the view of the radar beams, none of them could be associated with a simultaneous radar event.

It is shown that the part of the meteor population visible to the radars consists mainly of small particles in the $0.1-\mathrm{mm}$ radius range, and that the events observed the by optical system are caused by meteors from a different part of the size distribution, populated by $\mathrm{mm}$ sized particles. The agreement between the limiting radar magnitude estimated from the event rates and that estimated from the measured effective target crosssections, as well as the conspicuous absence of strong radar echoes, suggest that the scattering mechanism must display characteristics very similar to those proposed by Wannberg et al. (1996). A puzzling discrepancy between target velocities determined by time-of-flight methods and velocities determined from Doppler measurements indicates that the scattering may be from a part of the plasma cloud surrounding the meteor which is slipping relative to the parent particle. This will be the subject of continued investigation.

Acknowledgements. We gratefully acknowledge assistance of the EISCAT staff during the meteor experiment. The EISCAT Scientific Association is supported by the Centre National de la Recherche Scientifique of France, the Max-Planck-Gesellschaft of Germany, the Particle Physics and Astronomy Research Council of the United Kingdom, Norges Almenvitenskapelige Forskningsråd of Norway, Naturvetenskapliga Forskningsrådet of Sweden, Suomen
Akatemia of Finland, and the National Institute of Polar Research of Japan. The Swedish authors gratefully acknowledge financial support from the Naturvetenskapliga Forskningsrådet of Sweden.

Topical Editor D. Alcaydé thanks Q. Zhou and another referee for their help in evaluating this paper.

\section{References}

Brown, P., and J. Rendtel, 1993: Year of the Perseids, Sky and Telescope, 87, 24-37, 1994.

Bronshten, V. A., Physics of meteoric phenomena, D. Reidel, Dordrecht, 1983.

Chapin, E., and E. Kudeki, Radar interferometric studies of longduration meteor echoes observed at Jicamarca, J. Geophys. Res., 99, 8937-8949, 1994.

Clemesha, B. R., Sporadic neutral metal layers in the mesosphere and lower thermosphere, J. Atmos. Terr. Phys., 73, 725-736, 1995.

Clemesha, B. R., V. W. J. H. Kirchoff, D. M. Simonoch, and H. Takahashi, Evidence of an extraterrestrial source for the mesospheric sodium layer, Geophys. Res. Lett., 5, 873-876, 1978.

Evans, J. V., Theory and practice of ionosphere study by Thomson scatter radar, Proc. IEEE, 57, 496-530, 1969.

Evans, J. V., Radio-echo studies of meteors at 68-centimetre wavelength, J. Geophys. Res., 70, 5395-5416, 1965.

Evans, J. V., Radar observations of meteor deceleration, $J$. Geophys. Res., 71, 171-188, 1966.

Farley, D. T., The ionospheric plasma in Solar system plasma physics, vol. 3, ed: L. J. Lanzerotti, C. F. Kennel, and E. N. Parker, North Holland, New York, 1979.

German, M. J., and J. D. Mathews, Interference detection and correction applied to incoherent scatter radar power profile measurements, Radio Sci., 21, 745-751, 1986.

Hughes, D. W., Meteors, in J. A. M. McDonnel (ed), Cosmic Dust, chap. 3, Wiley, New York, 1978.

Jones, J. and A. R. Webster, Visual and radar studies of meteor head echoes, Planet Space Sci., 39, 873-878, 1991.

Malnes, E., N. Bjørnå, T. L. Hansen, Anomalous echoes observed with the EISCAT UHF radar at $100-\mathrm{km}$ altitude, Ann. Geophysicae, 14, 1328-1342, 1996.

Mathews, J. D., D. D. Meisel, K. P. Hunter, V. S. Getman, and Q. Zhou, Very high resolution studies of micrometers using the Arecibo 430 MHz radar, Icarus, 126, 157-169, 1997.

Pellinen-Wannberg, A., and G. Wannberg, Meteor observations with the European incoherent scatter. UHF radar, J. Geophys. Res., 99, 11379-11390, 1994.

Pellinen-Wannberg, A., and G. Wannberg, Enhanced ion-acoustic echoes from meteor trails, J. Atmos. Terr. Phys., 57, 495-506, 1996.

Rao, J., Storm Watch for the Perseids, Sky and Telescope, 86, 4348, 1993.

Sugar, R., Radio Propagation by Reflection from Meteor Trails, Proc IEEE, 52, 116-136, 1964.

Taylor, A. D., W. J. Baggaley, and D. I. Steel, Discovery of interstellar dust entering the Earth's atmosphere, Nature, 380, 323-325, 1996.

Wannberg, G., A. Pellinen-Wannberg, and A. Westman, An ambiguity-function-based method for analysis of Doppler decompressed radar signals applied to EISCAT measurements of oblique UHF/VHF meteor echoes, Radio Sci., 31, 497-518, 1996.

Westman, A., Development of high resolution radar measurements techniques for studies of transient phenomena in the ionospheric E and F layers, Doctoral Thesis, IRF Scientific Report 246, 1997.

Zhou, Q., and M. C. Kelley, Meteor observations by the Arecibo $430 \mathrm{MHz}$ incoherent scatter radar. 2. Results from timeresolved observations, J. Atmos. Terr. Phys., 59, 739-752, 1997. 
Zhou, Q., and J. D. Mathews, A spectral technique for the detection and correction of coherent interference effects on incoherent scatter radar power profiles, Radio Sci., 29, 11731177, 1994.
Zhou, Q., C. A. Tepley, and M. P. Sulzer, Meteor observations by the Arecibo $430 \mathrm{MHz}$ incoherent scatter radar, I. Results from time-integrated observations, J. Atmos. Terr. Phys., 57, 421431, 1995. 